\title{
INFLUENCE DE LA DENSITÉ INITIALE DE LARVES DE BROCHET (ESOX LUCIUS L., 1758) EN ÉLEVAGE INTENSIF SUR LA SURVIE, LA CROISSANCE ET LA CONSOMMATION JOURNALIĖRE DE NOURRITURE (ZOOPLANCTON, CHAOBORIDÉS).
}

\author{
P. GRES (1) ${ }^{*}$, P. LIM (2), A. BELAUD (2).
}

(1) Laboratoire de Zoologie-Protistologie, Équipe d'Hydrobiologie URA CNRS 138, 63177 Aubière Cedex, France.

(2) Laboratoire d'Ingénierie Agronomique, Équipe Environnement Aquatique et Aquaculture, E.N.S.A.T., 145 avenue de Muret, 31076 Toulouse Cedex, France.

\section{RÉSUMÉ}

Vingt mille larves de brochet (Esox lucius, L.) ont été élevées 35 jours en bac, avec une nourriture vivante (zooplancton et larves de Chaoboridés, Diptères) collectée en lagune d'épuration. Le but de cette expérience était de connaître l'influence de la densité initiale de stockage $\left(5000\right.$ à 20000 individus $/ \mathrm{m}^{3}$ ) sur la survie, la croissance et la quantité journalière de nourriture consommée au cours des phases zooplanctonophage et entomophage du stade larve en début d'alimentation exogène $(10-13 \mathrm{~mm}, \mathrm{LT})$ au stade juvénile de 50 à $60 \mathrm{~mm}$ (LT).

Les taux de survie, de cannibalisme et de croissance sont dépendants de la densité d'élevage. Le rythme alimentaire est monophasique et indépendant de la mise en charge et de la taille des brochetons. La consommation journalière, évoluant avec la taille du poisson, est peu influencée par la densité. Le taux d'évacuation gastrique est stable au cours de cet élevage. Le pourcentage du poids vif ingéré par jour par rapport au poids du poisson décroît du stade larve de $13-16 \mathrm{~mm}$ (30\%) au stade juvénile de $35-50 \mathrm{~mm}(20 \%)$. Des bases de rationnement pour une production de brochetons en élevage intensif sont proposées.

Mots-clés : brochet, larve, juvénile, survie, croissance, ration journalière, zooplancton, larves de Chaoborus.

\section{EFFECTS OF THE INITIAL STOCKING DENSITY OF LARVAL PIKES (ESOX LUCIUS L., 1758) ON SURVIVAL, GROWTH AND DAILY FOOD CONSUMPTION (ZOOPLANKTON, CHAOBORIDAE) IN INTENSIVE CULTURE.}

\section{ABSTRACT}

During 35 days, 20000 larval pikes (Esox lucius, L.) have been reared in tank and fed on natural live prey (zooplankton and Chaoboridae, Diptera) harvested in municipal sewage lagoon.

\footnotetext{
- Adresse actuelle : Fédération Départementale de la Loire des Associations de Pêche et de Protection du Milieu Aquatique. 24 rue d'Arcole, 42000 Saint-Étienne.
} 
The aim of this experimentation was to assess the effects of the initial stocking density (5000 to 20000 larvae $/ \mathrm{m}^{3}$ ) on the survival, the growth and the daily food intake from larvae at the onset of exogenous feeding $(10-13 \mathrm{~mm}$, LT) to $50-60 \mathrm{~mm}(\mathrm{LT})$ juveniles.

The survival, cannibalism and growth rates are density-dependent. There is a single daily feeding period, independently of stocking density and of pike size. Initial stocking density has little effect on the daily ration which increases with pike weight. Gastric evacuation rate is stable during the rearing period. The daily weight of fresh food ingested (in \%) decreases from larval stage $13-16 \mathrm{~mm}(30 \%)$ to juvenile stage $35-50 \mathrm{~mm}(20 \%)$. Basic live food rations for intensive culture of young pikes are proposed.

Key-words : pike, larvae, juveniles, survival, growth, daily ration, zooplankton, Chaoborus larvae.

\section{INTRODUCTION}

La première alimentation exogène des larves de brochet (Esox lucius, L.) est composée de proies zooplanctoniques vivantes (généralement, des entomostracés). La taille de ces proies ingérées en milieu naturel $s^{\prime}$ accroît rapidement pendant les deux à trois premières semaines (HUNT et CARBINE, 1951 ; FROST et KIPLING, 1967). Après cette phase zooplanctonophage stricte, les brochets recherchent et consomment des larves d'insectes (phase entomophage) (FRANKLIN et SMITH, 1963).

Les jeunes brochets s'attaquent ensuite à de jeunes poissons (phase ichtyophage), le cannibalisme pouvant rapidement se généraliser (CHODOROWSKA et CHODOROWSKI, 1975 ; BALVAY, 1983 ; BRY et al., 1995 ; GRES et BELAUD, 1995). Ces changements de phase progressifs se font en relation avec la disponibilité des différents types de proies dans le milieu (HUNT et CARBINE, 1951 ; FROST, 1954 ; BRY et al., 1995). L'évolution du rapport énergétique entre le gain métabolique dû à la proie et le coût de sa capture justifierait ces changements de comportement (NIKOLSKY, 1963 ; BALVAY, 1983).

Le comportement cannibale et le fait que les brochets ne puissent pas être facilement nourris sur proies inertes (type granulés) constituent les deux facteurs limitants majeurs de l'élevage intensif des jeunes brochets (ARRIGNON, 1976; HUET, 1976). Une importante disponibilité en macroinvertébrés, dans des milieux propices (petits étangs d'alevinage richement enherbés) (BRY et al., 1992), une faible densité de poissons (HORVATH, 1983), la présence de nombreuses caches (KLUPP, 1978 ; WRIGHT et GILES, 1987) et un spectre de taille de proies plus étendu (macrocrustacés, hirudinées, oligochètes, BALVAY, 1983) sont autant de facteurs en étang qui diffèrent l'apparition du cannibalisme. En élevage intensif, la disponibilité en proies de taille adaptée (imposant une source régulière et abondante de proies comme les lagunes d'épuration, BARNABÉ, 1979), la régulation de la température, le tri par taille (GRES et BELAUD, 1995), le contrôle de l'éclairement (ARRIGNON, 1976) permettent de retarder le déclenchement du comportement cannibale.

Bien que la littérature sur l'élevage et l'écologie du brochet soit très abondante (revues dans CROSSMAN et CASSELMAN, 1987 ; RAAT, 1988), il existe encore peu de données quantitatives sur les rations alimentaires, sur le taux de consommation journalière de nourriture, et sur les rythmes alimentaires des larves et juvéniles de brochet nourris de proies vivantes (BRY, 1992). Récemment, BRY et al. (1995) ont décrit les changements qualitatifs et quantitatifs de l'alimentation de jeunes brochets en conditions d'élevage extensif en étangs.

Le but de cet article est de compléter ces connaissances en se plaçant dans le contexte de l'élevage intensif, pratiqué en milieu contrôlé. II s'agit d'aborder la survie (et le cannibalisme), la croissance et la consommation journalière de nourriture de jeunes brochets élevés à différentes densités en circuit fermé et nourris avec des proies vivantes (zooplancton, puis larves de Chaoboridae, Diptères), récoltées en bassins d'épuration par lagunage naturel. 


\section{MATÉRIEL ET MÉTHODES}

L'élevage a été conduit à partir de brochets au stade embryon libre en fin de résorption de la vésicule vitelline (VR ${ }^{*}$ ) et il a duré cinq semaines (du 27 mars au 30 avril 1991). Il a été réalisé dans 16 bacs de 100 litres en circuit fermé d'eau (décantation, thermorégulation $\left(15^{\circ} \mathrm{C}\right.$ ), biofiltration et stérilisation Ultra-Violets), avec contrôles de la mortalité et de la croissance, d'une part, et analyses des contenus stomacaux prélevés au cours de cycles nycthéméraux, d'autre part (pour plus de détails : voir GRES, 1994).

\section{Caractéristiques environnementales de l'élevage}

La valeur de température, la concentration en oxygène dissous, la valeur de $\mathrm{pH}$ et la concentration en nitrites ont été contrôlées quotidiennement (à l'aide d'un oxy-thermomètre Y.S.I. 57, d'un pH-mètre Bioblock et d'un doseur rapide des nitrites Tetratest). La température moyenne sur la durée totale de l'expérience a été de $14,5 \pm 1,3^{\circ} \mathrm{C}$. La concentration moyenne en oxygène dissous a été de $8,8 \pm 0,5 \mathrm{mg} / \mathrm{L}$, soit $85 \%$ de la saturation à l'air ambiant. La valeur moyenne de $\mathrm{pH}$ a été de $7,3 \pm 0,4$ unités.

La concentration en nitrites, jusque-là inférieure à $0,1 \mathrm{mg} \mathrm{N} / \mathrm{L}$, a augmenté entre le $11 \mathrm{e}$ et le $20 \mathrm{e}$ jour $(0,25$ à $0,50 \mathrm{mg} \mathrm{N} / \mathrm{L})$. Ces concentrations plus élevées ne peuvent en aucun cas avoir eu un effet direct sur la survie des larves de brochet, en raison des concentrations élevées en oxygène et du faible temps d'exposition (DE KINKELIN et al., 1985). Cependant, par précaution, il a été décidé un renouvellement total de la masse d'eau durant les $17 \mathrm{e}$, $18 \mathrm{e}$ et $19 \mathrm{e}$ jours. La baisse consécutive de la température a conduit à réduire l'activité alimentaire des larves de brochets durant la troisième semaine d'élevage. Les résultats du 21 e jour seront donc à interpréter avec prudence. En raison de mortalités plus importantes dans les bacs de densité D4, nous n'avons pas réalisé de prélèvements aux $28 \mathrm{e}$ et $35 \mathrm{e}$ jours.

Le mode d'éclairement (9 à 11 heures par jour) et les traitements prophylactiques sont décrits en détail dans GRES (1994).

\section{Mise en charge et échantillonnage}

Quatre lots avec quatre réplicats ont correspondu à des densités initiales de mise en charge (D, exprimée en VR/m ${ }^{3}$ ) croissantes : $D 1=5000 \mathrm{VR} / \mathrm{m}^{3}$ (soit $500 \mathrm{VR}$, pour les bacs $B 1$ à B4), D2 $=10000 \mathrm{VR} / \mathrm{m}^{3}$ (soit $1000 \mathrm{VR}$, pour les bacs B5 à B8), D3 $=15000 \mathrm{VR} / \mathrm{m}^{3}$ (soit $1500 \mathrm{VR}$, pour les bacs $B 9$ à $B 12$, à l'exception de $B 10$ qui n'a reçu que $1350 \mathrm{VR}$ ) et $\mathrm{D} 4=20000 \mathrm{VR} / \mathrm{m}^{3}$ (soit 2000 VR, pour les bacs B13 à B16).

Après cette répartition, le jour 0 a été calé sur l'observation de la prédation active de $80 \%$ des individus d'un échantillon de 50 VR (attitude typique en S, d'après BRAUM, 1964). L'activité alimentaire des embryons libres a commencé le même jour dans tous les bacs, indépendamment des densités imposées.

Les échantillonnages de jeunes brochets ont été effectués aux 7e, 14e, 21e, 28e et 35e jours d'élevage dans les quatre mêmes bacs de densités différentes: B1, B5, B9 et B13. Les poissons prélevés (conservés dans une solution de formaldéhyde $10 \%$ ) n'ont pas été remplacés, mais le volume des bacs d'échantillonnage a été réajusté (baisse de niveau) afin de maintenir la valeur de la densité initiale. Cinq poissons par bac ont été prélevés, au hasard, à 09h, 12h, 15h, $18 \mathrm{~h}, 21 \mathrm{~h}, 24 \mathrm{~h}, 03 \mathrm{~h}, 06 \mathrm{~h}$ et $09 \mathrm{~h}$, soit 45 poissons par cycle nycthéméral et par bac. Au total, 907 estomacs de brochets ont été examinés. Le prélèvement de 9 heures était effectué avant le début de la distribution de nourriture.

\section{Proies vivantes, collecte et distribution}

La nourriture était récoltée, à l'aide de filets et de collecteurs électriques (voir détails dans GRES, 1994), dans les bassins de lagunage naturel (milieu particulièrement producteur en invertébrés, BARNABÉ, 1979 ; GUERRIN, 1990 ; DURIEU, 1995) de l'agglomération de Baraqueville (Aveyron) et sur deux lagunages proches.

\footnotetext{
- Les stades de développement des brochets ont été codifiés suivant la classification de BALON (1975, in BRY et al., 1991): larve ( $L T=12$ à $35 \mathrm{~mm}$ ) et juvénile ( $L T=35$ à $70 \mathrm{~mm}$ ).
} 
Pendant les 12 premiers jours d'élevage (Tableau I), un régime zooplanctonophage strict a été respecté. Les fractions de démarrage (du 1er au 3e jour) étaient comprises entre 300 et $500 \mathrm{~mm}$, et essentiellement constituées de petits Cladocères (Chydorus sphaericus, Bosmina longirostris, Daphnia sp.), selon les recommandations de DROST (1987) et BRY (1992) qui avaient observé respectivement une meilleure ingestion et une meilieure survie ultérieure avec ce type de proies. A partir du $4 \mathrm{e}$ jour, les fractions zooplanctoniques déversées étaient celles retenues par un tamis de $500 \mathrm{~mm}$ (Copépodes Cyclopoidés et Daphnia sp.).

\section{Tableau I : Diagramme décrivant les apports journaliers de nourriture vivante (PV \% = pourcentage du poids vif).}

Table I : Diagram illustrating daily live food supply (PV \% = percentage of body weight).

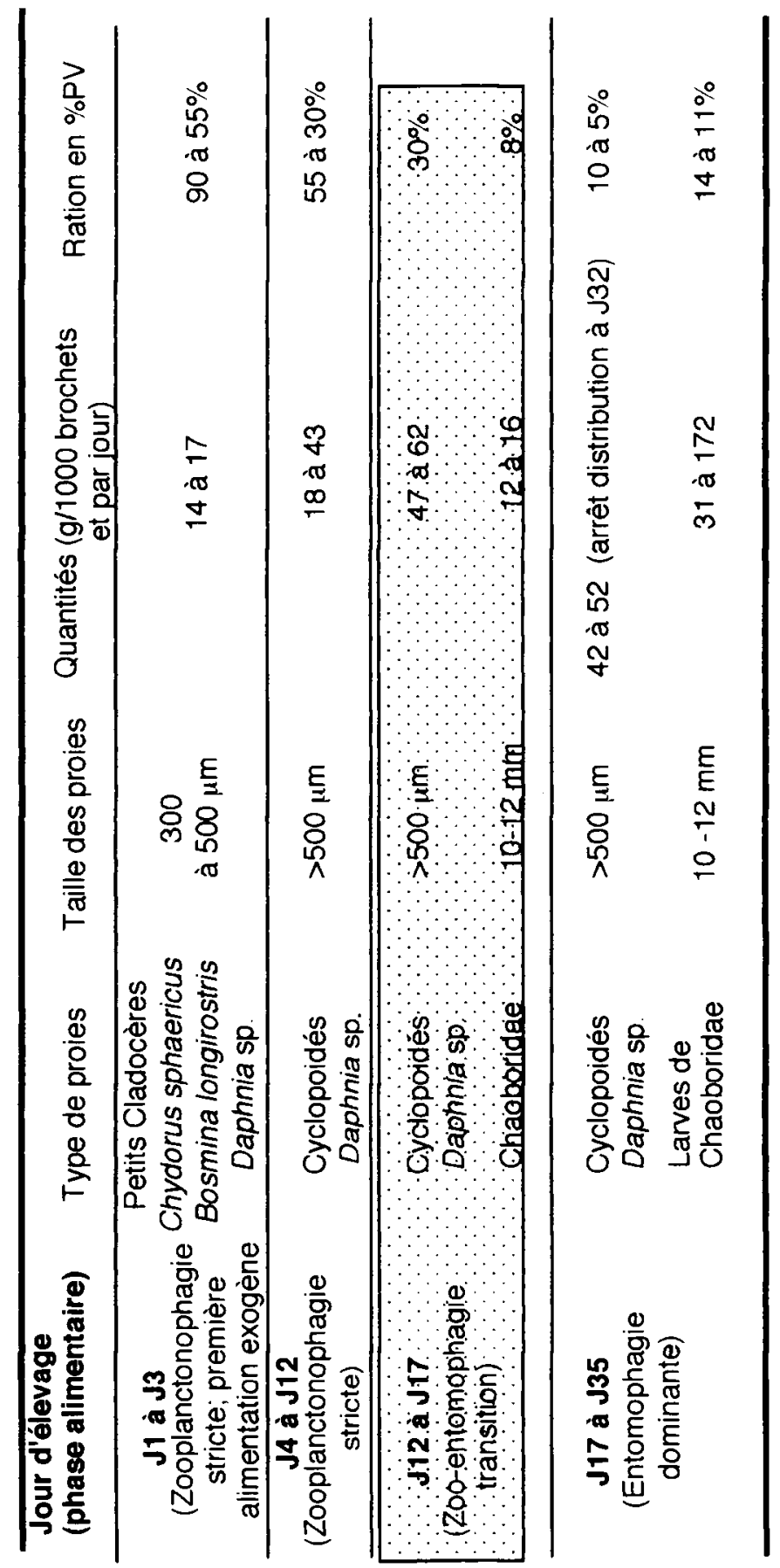


Après le 12e jour d'élevage, le régime a été de type zoo-entomophage. Durant cette phase, des larves de Diptères (stades III et IV de Chaoboridae, Chaoborus flavicans, d'après DURIEU, 1995) ont été ajoutées; la part relative du zooplancton dans la ration distribuée diminuant progressivement en cours d'élevage (arrêt au 32e jour) au profit des larves d'insectes.

Les rations distribuées ont été ajustées par rapport au poids vif des brochetons, en se basant sur des prévisions de croissance théorique et sur un rationnement conforme à la fois aux résultats d'une expérimentation préliminaire (GRES, 1994) et aux données de la littérature (SMISEK, 1968 ; LANE et JACKSON, 1969 ; LUQUET et LUQUET, 1983 ; KAUSHIK et al., 1985 ; LEJOLIVET, 1988). Une quantité moyenne de 40 à $25 \%$ du poids vif des brochets, du début à la fin de l'élevage, a été fournie. D'un point de vue nombre de proies disponibles par poisson, les larves et les juvéniles de brochets disposaient de 750 à 500 zooplanctontes et de 3 à 38 Chaoborus par jour, du début à la fin de l'élevage. Le critère du groupement des poissons en essaim a été adopté pour juger de la suffissance des proies disponibles (GUERRIN, 1983 ; GRES, 1994).

\section{Mesures sur les poissons prélevés}

Chaque poisson a été mesuré (longueur totale, LT) à $0,5 \mathrm{~mm}$ près et pesé à $0,1 \mathrm{mg}$ près (poids total, Pt). Nous les avons ensuite regroupés en 5 fourchettes " indicatives " de taille correspondant aux 5 dates de prélèvement $(13,5-16,5 \mathrm{~mm} ; 16,5-20 \mathrm{~mm} ; 20-25 \mathrm{~mm} ; 25-35 \mathrm{~mm}$; 35-50 mm). L'estomac a été prélevé et le contenu dégagé de la paroi stomacale. Après suppression de l'excès d'eau sur papier cellulose, la quantité de proies ingérées a été pesée à $0,1 \mathrm{mg}$ près (balance Oerlikon précisa 125). Le contenu a été aussitôt placé dans une cellule de Sedgewick Rafter pour observation sous loupe binoculaire (grossissement 7 à 40 fois).

Les organismes ingérés ont été comptés et identifiés, et les éléments les moins détériorés (20 à 50 individus pour le zooplancton) ont été mesurés à $25 \mu \mathrm{m}$ près, suivant la méthode définie par CULVER et al. (1985).

\section{Traitement des données}

\section{Survie}

Connaissant le nombre d'embryons libres en fin de résorption (VR) mis en charge initialement $\left(\mathrm{N}_{\mathrm{i}}\right)$, le nombre de poissons prélevés (ainsi que les poissons malingres ou " fils" retirés des bacs) $\left(\mathrm{N}_{\mathrm{pp}}\right)$ pour l'expérimentation, le nombre de poissons morts $\left(\mathrm{N}_{\mathrm{m}}\right)$ et le nombre de poissons vivants obtenus au $28 \mathrm{e}$ jour $\left(\mathrm{N}_{\mathrm{v}}\right)$, nous avons calculé le nombre de brochets disparus (cannibalisme, $\mathrm{N}_{\mathrm{C}}$ ):

$$
\mathbf{N}_{\mathbf{c}}=\mathbf{N}_{\mathrm{i}}-\left(\mathbf{N}_{\mathrm{pp}}+\mathbf{N}_{\mathbf{m}}+\mathbf{N}_{\mathbf{v}}\right)
$$

Les valeurs $N_{v}, N_{m}$ et $N_{c}$, rapportées à $N_{i}-N_{p p}$, fournissent le taux de survie, de mortalité naturelle et de mortalité par cannibalisme.

\section{Croissance}

Pour chaque prélèvement et chaque densité, les valeurs de longueurs totales (LT, mm) et de poids frais totaux (Pt, $\mathrm{mg}$ ) des poissons, minimales ( $L T m$ et $P t m$ ), maximales (LTM et PtM) et moyennes (XLT et XPT) ont été déterminées. Le taux relatif de croissance pondérale (TRC) a été calculé selon la relation :

$$
\operatorname{TRC}(\%)=\frac{\mathrm{Pt}_{\mathbf{2}}-\mathrm{Pt}_{1}}{\mathrm{Pt}_{\mathbf{1}}} \times 100
$$

où $\mathrm{Pt}_{2}=$ poids du poisson au temps $\mathrm{t}_{2}$;

$\mathrm{Pt}_{1}=$ poids du poisson au temps $\mathrm{t}_{1}$.

Le taux spécifique de croissance (TSC) entre le jour $0\left(t_{0}\right)$ et le jour $35\left(t_{35}\right)$ était :

$$
T S C=100 \times \frac{\left(\operatorname{lnPt_{0}}-\operatorname{lnPt_{35}}\right)}{t_{0}-t_{35}}
$$




\section{Régime alimentaire}

Le régime alimentaire a été chiffré de deux manières :

- par la contribution numérique ( $N$ \%) qui correspond au rapport entre le nombre d'individus de chaque type de proie considérée et le nombre total d'organismes contenus dans l'estomac ; la formule :

- et par l'indice alimentaire (IA) de LAUZANNE (1975) des différents types de proies suivant

$$
I A=\frac{(F \% \times W \%)}{100}
$$

où $\mathrm{F} \%$ est l'indice de fréquence de la proie considérée (proportion d'estomacs contenant cette proie par rapport à l'ensemble des contenus stomacaux pleins examinés) et $W \%$ est la contribution pondérale de chaque type de proie par rapport au poids total du contenu stomacal.

La longueur moyenne des proies ingérées a été calculée pour chaque estomac examiné, puis une moyenne globale $\left(L_{t p i}\right.$, en $\mu \mathrm{m}$ ou $\left.\mathrm{mm}\right)$ a été étendue à chaque prélèvement.

\section{Sélection des proies}

A partir de l'examen des échantillons de la nourriture vivante distribuée (pourcentage relatif des différentes espèces-proies) et de la contribution numérique ( $N \%)$ des espèces-proies ingérées, l'indice d'électivité d'IVLEV (1961) a été obtenu par la formule :

$$
E=\frac{(r i-p i)}{(r i+p i)}
$$

où $\mathrm{ri}=$ proportion de la proie $\mathrm{i}$ dans l'estomac $(=\mathrm{N} \%)$;

pi $=$ proportion de la proie i dans le milieu (eau d'élevage).

E varie de -1 à +1 . La valeur -1 exprime un évitement total de la proie par le prédateur, la valeur nulle : une sélection passive, et la valeur +1 : une sélection active et exclusive de la proie.

Quantités de proies consommées et rythme alimentaire

A partir de la longueur moyenne de chaque type de proie ingérée, nous avons obtenu le poids sec ingéré correspondant, en utilisant les relations poids - longueur $W=a \times L^{b}$ de DUMONT et al. (1975) pour le zooplancton et DUMONT et BALVAY (1979) pour les larves de Chaoboridés, puis nous l'avons converti en poids frais selon le pourcentage d'humidité de chaque type de proie. En cumulant les poids des différents items, nous avons établi le poids frais du contenu stomacal (PCS) (GRES, 1994). Cette méthode " additive " est validée par le fait que les valeurs ainsi calculées sont corrélées positivement de façon hautement significative $(r=0,94$; $p=0,0001 ; N=132$ ) à la pesée du contenu stomacal brut (brochets du bac B1). Le traitement de tous les poissons prélevés a conduit à établir le poids frais moyen du contenu stomacal pour chaque bac (donc les différentes densités d'élevage) et à chaque stade de l'élevage.

Le coefficient de réplétion ( $\mathrm{Cr} \%=$ Poids frais du contenu stomacal/poids frais du brocheton $\times 100$ ) a été calculé pour chaque poisson sacrifié et les moyennes établies pour chaque heure de prélèvement, les différentes densités d'élevage et chaque stade.

Nous avons utilisé le logiciel MAXIMS (JARRE et al., 1990 ; PALOMARES, 1991) pour estimer la quantité quotidienne de nourriture consommée $\left(R_{j}\right.$, en $\mathrm{mg}$ ) par les poissons à partir des pointages trihoraires des contenus stomacaux. 
Le taux de consommation journalière ( $\mathrm{Cpv}$, en \%) est la quantité quotidienne de nourriture consommée rapportée au poids total moyen (XPT) des brochets suivant la relation :

$$
\mathrm{Cpv}=\frac{\mathbf{R}_{\mathrm{j}} \times 100}{\mathrm{XPT}}
$$

L'outil MAXIMS permet de tracer le rythme alimentaire et, en particulier, de voir si la vitesse d'ingestion est constante pendant la phase de nutrition ou si elle décroît lorsque l'estomac est distendu.

La durée totale de l'évacuation gastrique a été calculée comme le temps s'écoulant entre l'heure de fin d'alimentation et le moment où les coefficients de réplétion moyens deviennent inférieurs à $0,5 \%$.

Le nombre moyen de proies de type i ingérées $\left(N_{\mathrm{mp}}\right)$ en 24 heures a été évalué par la formule suivante :

$$
N_{\text {mpi }}=\frac{T_{p i}}{p_{p i}}
$$

où

$--->T p_{i}=$ poids total de toutes les proies $\mathrm{i}$ ingérées :

$$
T p i=\frac{W \% \times R_{j}}{100}
$$

(W \% est la contribution pondérale de la proie i par rapport au poids total du contenu stomacal, et $R_{j}(\mathrm{mg})$ est la ration journalière) ;

$-->p_{p i}(m g)$ est le poids frais moyen de la proie $\mathrm{i}$ ingérée.

\section{Analyses statistiques}

Des analyses de variance (ANOVA) à un facteur simple ou à deux facteurs ont été réalisées pour déterminer l'effet de la densité des poissons en élevage sur la croissance, la survie, le cannibalisme, les quantités journalières ingérées. Des régressions simples ont été utilisées pour examiner les relations entre le poids calculé et le poids mesuré des contenus stomacaux, et entre la densité et les taux de survie et de cannibalisme.

\section{RÉSULTATS} (Tableau III)

Taux de survie, de mortalité (Tableau II) liée au cannibalisme et croissance

Les taux de survie à 28 jours (44 à $69 \%$ ) s'avèrent inversement proportionnels à la mise en charge initiale $(r=0,83 ; p=0,0001 ; 13 \mathrm{ddl})$. Une ANOVA indique que la survie a été densité dépendante $(F=8,17 ; p=0,0031)$. Le taux de cannibalisme est corrélé positivement et de manière hautement significative avec la mise en charge $(r=0,68 ; p=0,0056 ; 13 d d l)$. Une ANOVA révèle que le cannibalisme a été densité dépendant $(F=4,97 ; p=0,018)$. La variabilité inter-bacs diminue avec l'augmentation de la densité initiale de mise en charge (respectivement 29, 26, 10 et $7 \%$ pour D1, D2, D3 et D4). 
Tableau II : Bilan au jour 28 du nombre de brochets vivants $\left(\mathbf{N}_{\mathbf{v}}\right)$, morts $\left(\mathbf{N}_{\mathbf{m}}\right)$, victimes du cannibalisme $\left(N_{c}\right)$ et taux de survie (S \%), de mortalité naturelle ( $M \%$ ) et de cannibalisme ( $\left.C \%\right)$ (et écart type) sous des conditions initiales croissantes de mise en charge $\left(\mathbf{N}_{\mathbf{i}}=\right.$ nombre initial de V.R. ; $\mathbf{N}_{\mathrm{pp}}=$ nombre de poissons prélevés).

( $\left.{ }^{\star}\right)$ Transfert : au début de l'élevage, les larves malingres, qui ne se nourrissaient pas, ont été retirées des bacs suivant les recommandations de PECOR (1978) et LAPLANTE (1988).

Table II : Assessment at day 28 of the number of surviving $\left(N_{v}\right)$, dead $\left(N_{m}\right)$, cannibalized $\left(\mathbf{N}_{c}\right)$ young pikes and of survival (S \%), natural mortality ( $M \%$ ) and cannibalism (C \%) rates (and standard deviation) of pikes under increasing initial stocking density $\left(N_{i}=\right.$ initial number of V.R. ; $\mathbf{N}_{\mathbf{p p}}=$ number of sampled pikes).

( $\left.{ }^{*}\right)$ Transfer : at the beginning of the rearing period, unfed pinhead fry have been removed from tanks as suggested by PECOR (1978) and LAPLANTE (1988).

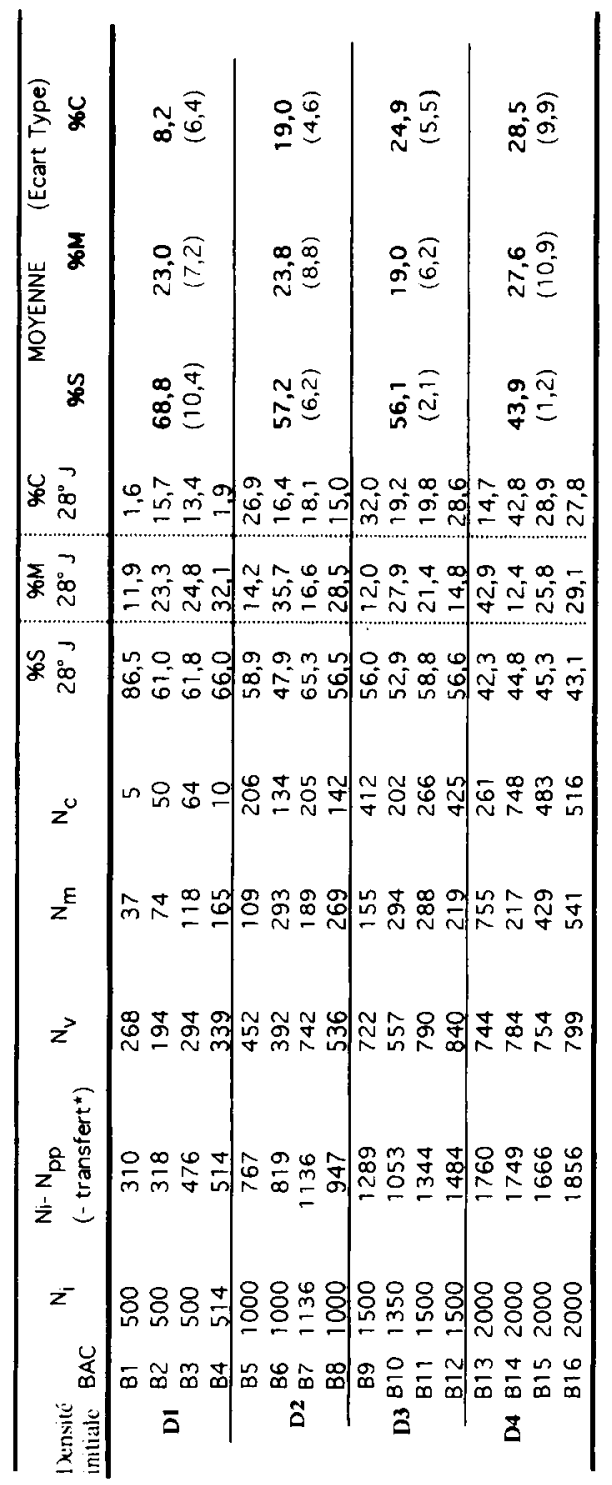

Les tailles atteintes au 35e jour sont comprises entre 29 et $54 \mathrm{~mm}$, avec des moyennes par bac s'échelonnant entre 37 et $43 \mathrm{~mm}$. Les taux spécifiques de croissance entre 0 et 35 jours sont légèrement plus élevés dans les bacs les moins chargés (TSC, de $t=0$ à $t=35: 11,2 \%$ pour la densité D1, 10,4\% pour la densité D2 et 9,9\% pour la densité D3). 
Tableau III : Croissances linéaire $(\mathrm{mm})$ et pondérale $(\mathrm{mg})$ des brochets élevés sous des densités initiales croissantes pendant 35 jours (LT, LTm, LTM, XLT = longueurs totale, minimum, maximum, moyenne; Pt, Ptm, PtM, XPT = poids total, minimum, maximum, moyen ; V.R. = embryon libre en fin de résorption de vésicule vitelline; TRC = taux relatif de croissance, $\% ;$ TSC = taux spécifique de croissance, $\% ; \mathbf{N}=$ nombre de poissons analysés).

Table III : Growth in length $(\mathrm{mm})$ and weight $(\mathrm{mg})$ of pikes under increasing initial stocking density during 35 days (LT, LTm, LTM, XLT = total length, minimum, maximum, mean ; Pt, Ptm, PtM, XPT = total weight, minimum, maximum, mean ; V.R. = resorbed yolk sac free embryo ; TRC = relative growth rate, $\%$; TSC $=$ specific growth rate, $\% ; N=$ number of analysed fishes).

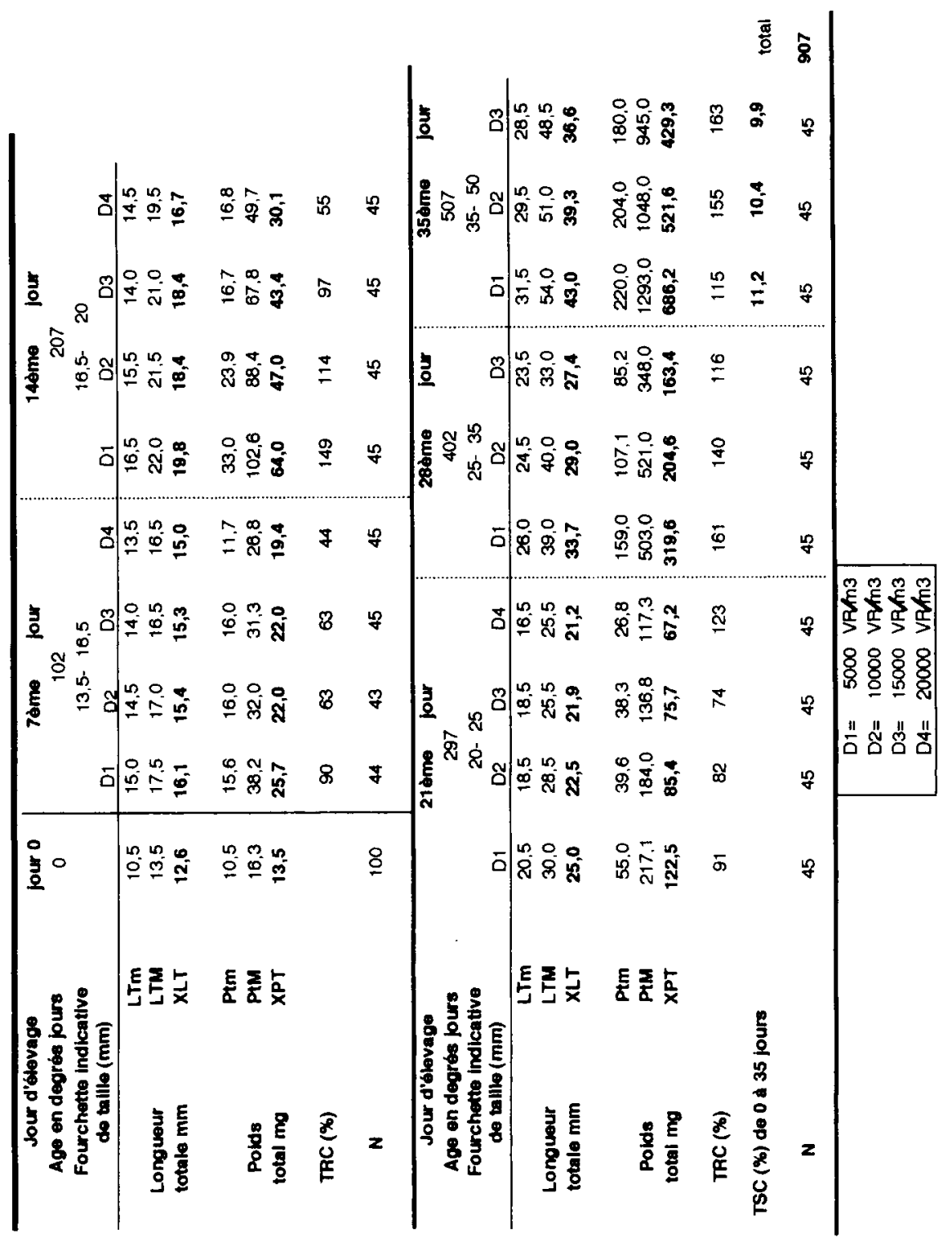

En ce qui concerne les taux relatifs de croissance (TRC), on ne relève aucun effet de la densité (ANOVA, $F=2,06$ et $p=0,18$ ). L'incident de fonctionnement du circuit lors de la $3 e$ semaine d'élevage a eu pour effet de diminuer anormalement le taux relatif de croissance par rapport aux $2 \mathrm{e}$ et $4 \mathrm{e}$ semaines, sauf pour le bac B13 à forte mise en charge (D4) où l'on observe une augmentation. 


\section{Régime alimentaire, nombre de proies ingérées et sélection des proies (figure 1 ; tableaux (Va et IVb)}

Les larves de brochet de 13,5-16,5 mm consomment sans sélection apparente $(E=-0,06$ à -0,31) entre 20 et 65 Cyclopoidés par jour. Ceux-ci représentent les proies dominantes, à la fois de l'indice alimentaire (IA de 75 à $94 \%$ ) et des rations déversées (82\%). On observe une sélection moyenne à active sur Bosmina longirostris et Chydorus sphaericus $(+0,40$ à $+0,92)$.

A compter de l'apport de larves de Chaoboridés à partir du $12 \mathrm{e}$ jour, ces proies prennent une part prépondérante (IA moyen $76 \%$ ) dans I'alimentation, avec un nombre moyen de proies consommées par jour et par brochet passant de 1 (au stade larve 16,5-20 mm) à 37 Chaoborus (au stade juvénile 35-50 mm). Les larves et les juvéniles de brochet continuent à consommer sans sélection active des proies zooplanctoniques (de 8 à 400 par individu et par jour). II s'agit surtout des Cyclopoidés, ces derniers faisant l'objet d'une sélection plus active par les poissons plus grands.

Les valeurs élevées et positives des indices d'électivité pour Chydorus, Alona et Daphnia ne sont pas déterminantes, car ces proies sont très accessoires. Nous considérons que l'alimentation des larves de brochet est le plus souvent opportuniste, dans cet élevage expérimental.

En ce qui concerne les proies essentielles (Cyclopoidés, Bosmina, Chaoborus), il n'existe pas d'effet de la densité sur le choix de ces proies (ANOVA, respectivement $p=0,95 ; p=0,19$ et $p>0,09$ pour IA), mais il apparaît par contre une relation taille de proie-taille brochet (ANOVA, respectivement $p=0,03 ; p=0,005$ et $p=0,0001$ ).

\section{Rythme alimentaire des brochetons}

Les rythmes alimentaires des brochets, représentés par l'évolution horaire du coefficient de réplétion (figure 2), sont, à fréquences pratiquement égales, mono ou biphasiques. Pourtant, nos observations directes du comportement des brochets indiquent chaque jour une activité de chasse biphasique (succession d'une activité prédatrice intense, d'une phase de "repos ", puis d'une reprise de l'activité alimentaire; GRES, 1994). L'ajustement des différents cas expérimentaux avec le modèle MAXIMS conduit à déterminer une seule phase d'alimentation dans quasiment tous les cas (tableau $\mathrm{V}, 17$ cas sur 18 ).

\section{Consommation journalière de nourriture}

II n'existe pas de différence significative entre les rations consommées par les brochets soumis aux différentes densités initiales de mises en charge (ANOVA, $p>0,05$ ), mais les rations ingérées sont étroitement corrélées avec le poids des brochets $(r=0,97 ; p=0,0001)$, ceci indépendamment des densités (ANOVA, $p>0,05$ ).

Les brochets consomment entre 3 et $150 \mathrm{mg}$ de poids frais de proies (tableau $\mathrm{V}$ ) du stade $13 \mathrm{~mm}$ à $50 \mathrm{~mm}$ durant les 9 à 11 heures d'activité alimentaire correspondant à la phase éclairée du nycthémère, soit un $\mathrm{Cpv} \%$ compris entre 6 et $30 \%$. La baisse de la ration consommée au jour 21 correspond aux problèmes thermiques de la troisième semaine.

Les durees moyennes d'évacuation gastrique sont comprises entre 10 et 15 heures pour toutes les séries d'observations confondues, sauf pour les larves de $16,5-20 \mathrm{~mm}$, qui requièrent entre 15 et 18 heures pour vider leur estomac.

\section{DISCUSSION}

\section{Comparaison des performances de l'élevage avec celles de la littérature}

Les taux de survie à 28 jours (44 à $69 \%$ ) sont, en élevage sous fortes densités, inversement proportionnels à la densité initiale. Les données de WRIGHT et GILES (1987) concluaient à une survie indépendante de la densité durant les 21 premiers jours d'élevage (en étang), puis à une relation densité dépendante après l'instauration du cannibalisme. Les conditions d'élevage totalement différentes ne permettent pas de comparer directement les deux ensembles de 


\section{Larves de Chaoboridés \\ $\square$ Cladoceres (Bosmina, Chydorus, Alona, Daphnia) \\ Cyclopoidés}

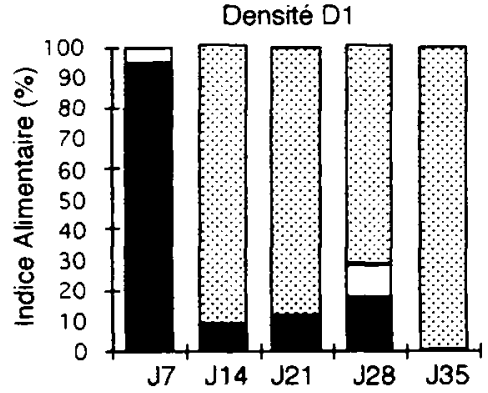

$1 \mathrm{~A} \%$
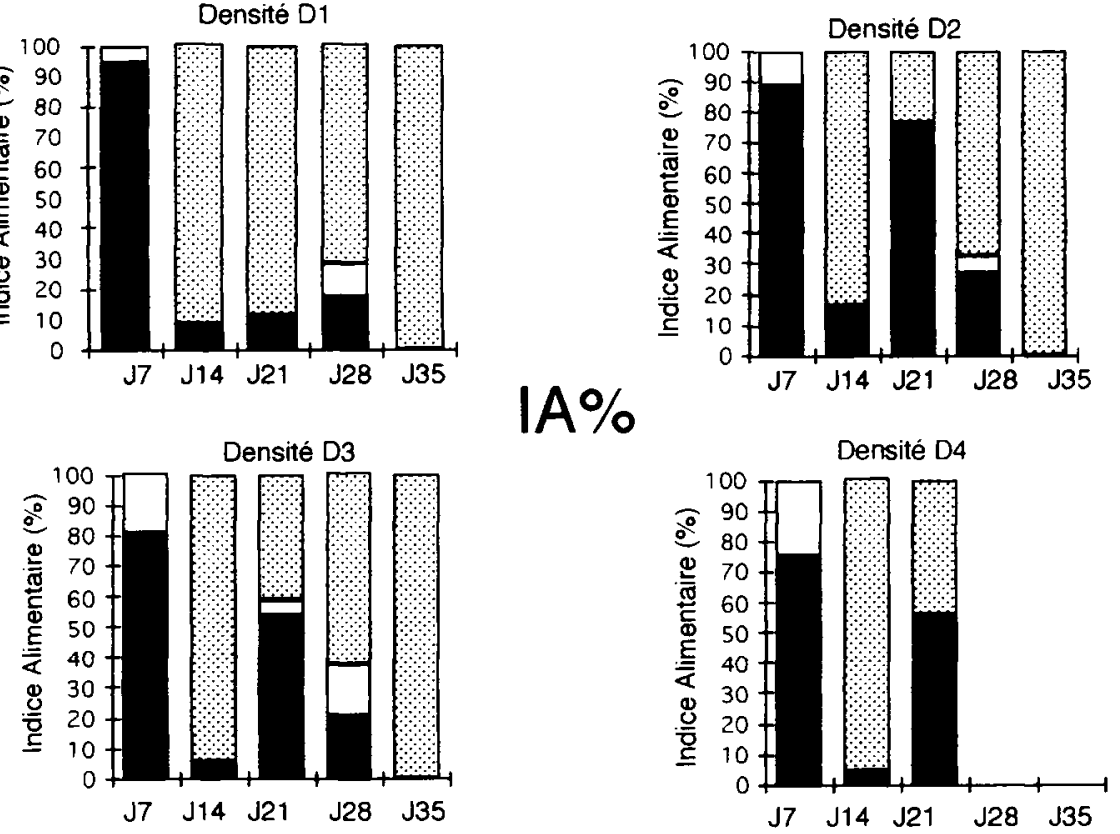

Densité D1

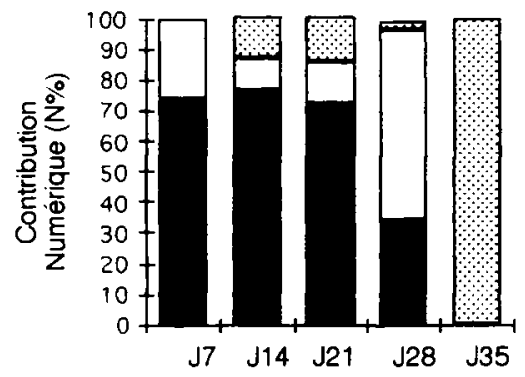

Densité D2
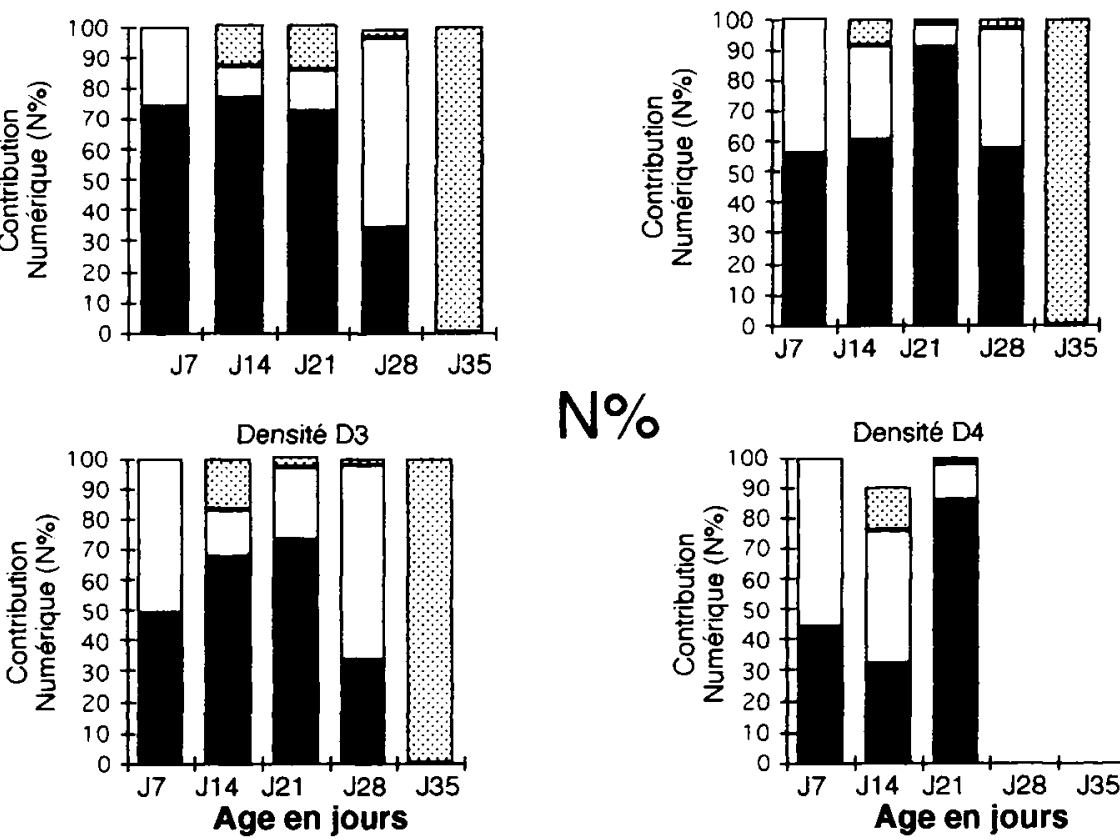

$\mathrm{N} \%$

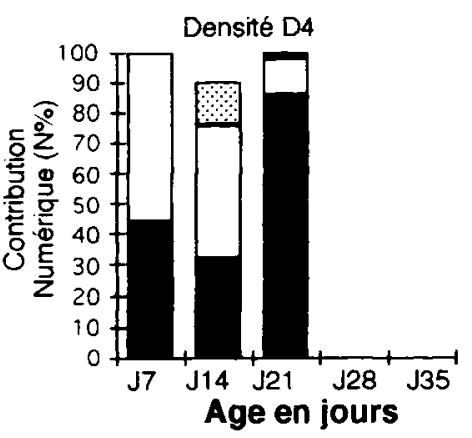

Figure 1 : Evolution temporelle de l'Indice Alimentaire (IA, \%) et de la Contribution Numérique (N, \%) des espèces-proies ingérées en 24 heures par différentes classes d'âge de brochets élevés sous des densités initiales croissantes.

Figure 1 : Temporal shift of dietary index (IA, \%) and numerical contribution (N, \%) of preys ingested over a 24 hours period by young pikes, according to pike size and to initial stocking density. 
Tableau IV : (a) - Longueur totale moyenne $\left(L_{t p i}, \mu \mathrm{m}\right.$ et $\mathrm{mm}$ ), nombre moyen $\left(\mathrm{N}_{\mathrm{mpi}}\right)$ et indice d'électivité (E) des espèces-proies ingérées en 24 heures par différentes classes de taille de brochetons élevés sous des densités initiales croissantes.

(b) - Composition qualitative (\%) du zooplancton disponible (7ème, 14ème, 21ème et 28ème jours).

Table IV : (a) - Mean total length ( $L_{t p i}, \mu \mathrm{m}$ and $\left.\mathbf{m m}\right)$, mean number $\left(N_{m p i}\right)$ and electivity index $(E)$ of preys ingested over a 24 hours period by young pikes, according to pike size and to initial stocking density.

(b) - Numerical composition (\%) of available zooplankton (7th, 14th, 21st and 28th days).
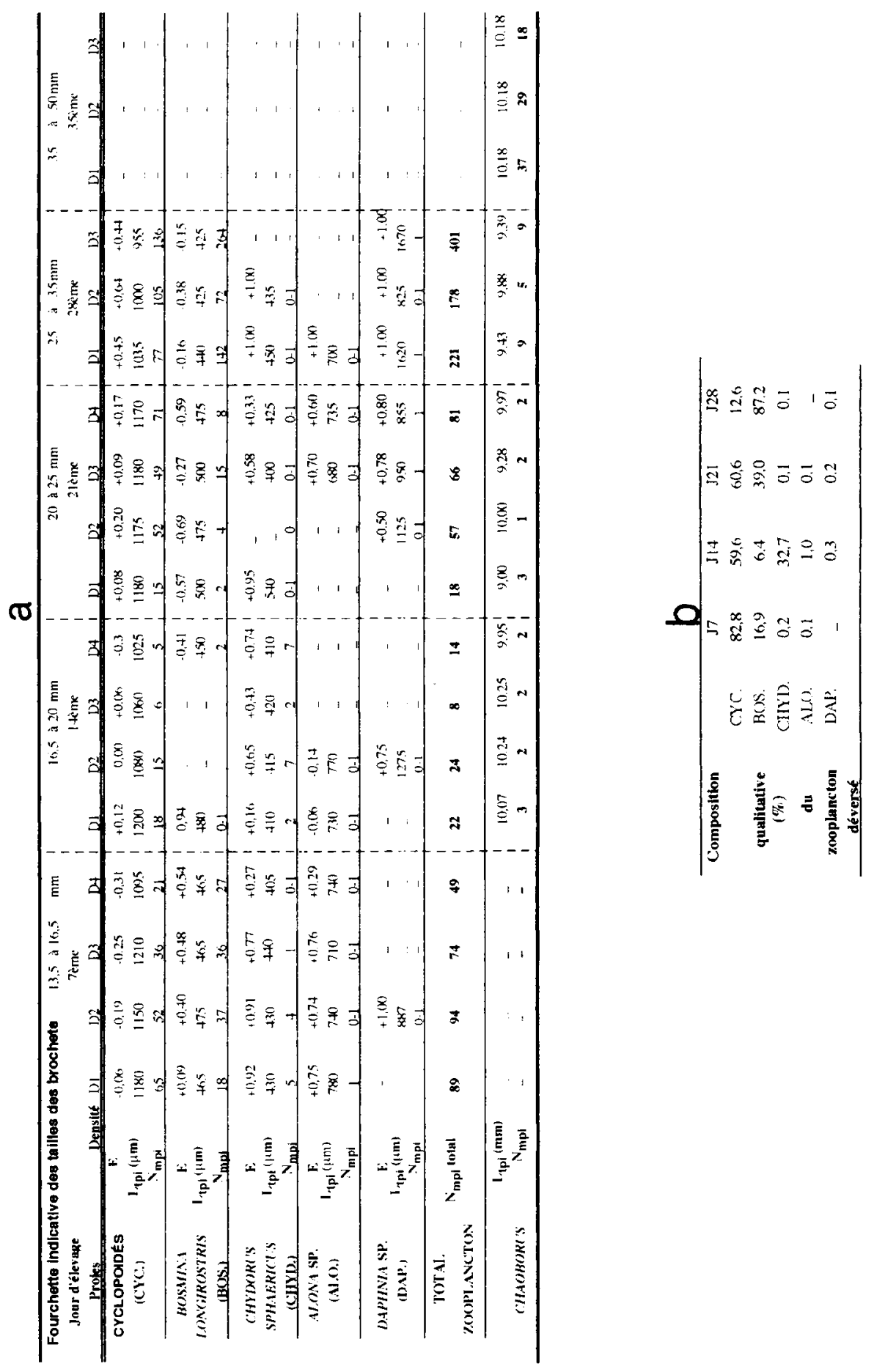

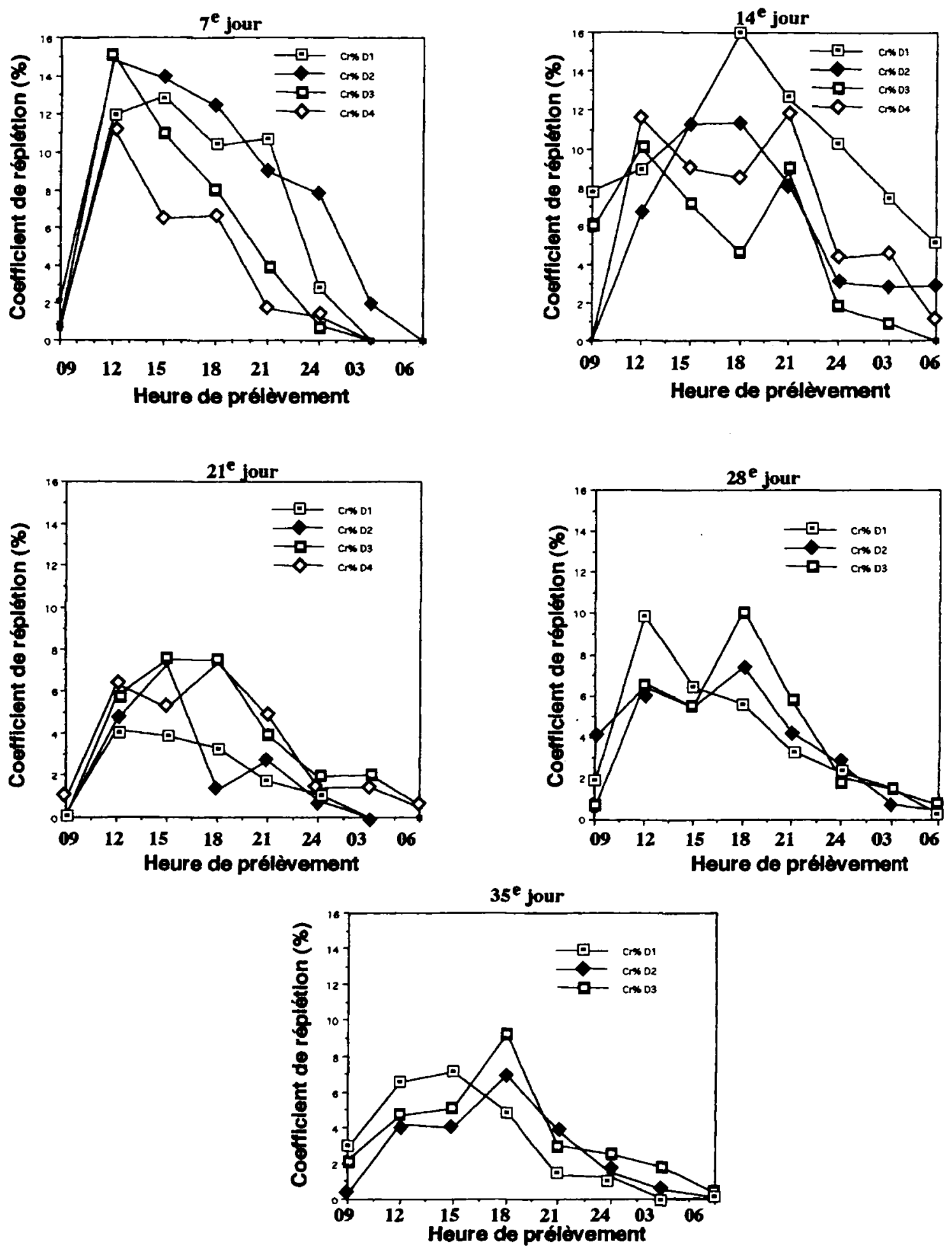

Figure 2 : Evolution sur une période de 24 heures du coefficient de réplétion ( $\mathrm{Cr}, \%)$ des larves et des juvéniles de brochet en fonction de leur âge $(7$ à 35 jours depuis le début de l'alimentation exogène) et de la densité initiale de mise en charge (D1 $=5000 \mathrm{VR} / \mathrm{m}^{3}$ à D4 $=20000 \mathrm{VR} / \mathrm{m}^{3}$.

Figure 2 : 24 hours profiles of repletion coefficient $(\mathrm{Cr}, \%)$ in pike larvae and juveniles : effect of age since first exogenous feeding ( 7 to 35 days) and of initial stocking density (from D1 $=5000$ $\mathrm{fry} / \mathrm{m}^{3}$ to $\mathrm{D4}=20000 \mathrm{fry} / \mathrm{m}^{3}$ ). 
Tableau V : Ration journalière ( $\mathrm{mg}$ ), durée de l'évacuation gastrique (h) et pourcentage de poids vif ingéré par jour (Cpv \%) pour des classes de taille croissante de brochetons élevés à différentes densités.

Table V : Daily food intake ( $\mathrm{mg})$, duration of gastric evacuation (h) and percentage of body weight ingested per day (Cpv \%), according to pike size and to initial stocking density.

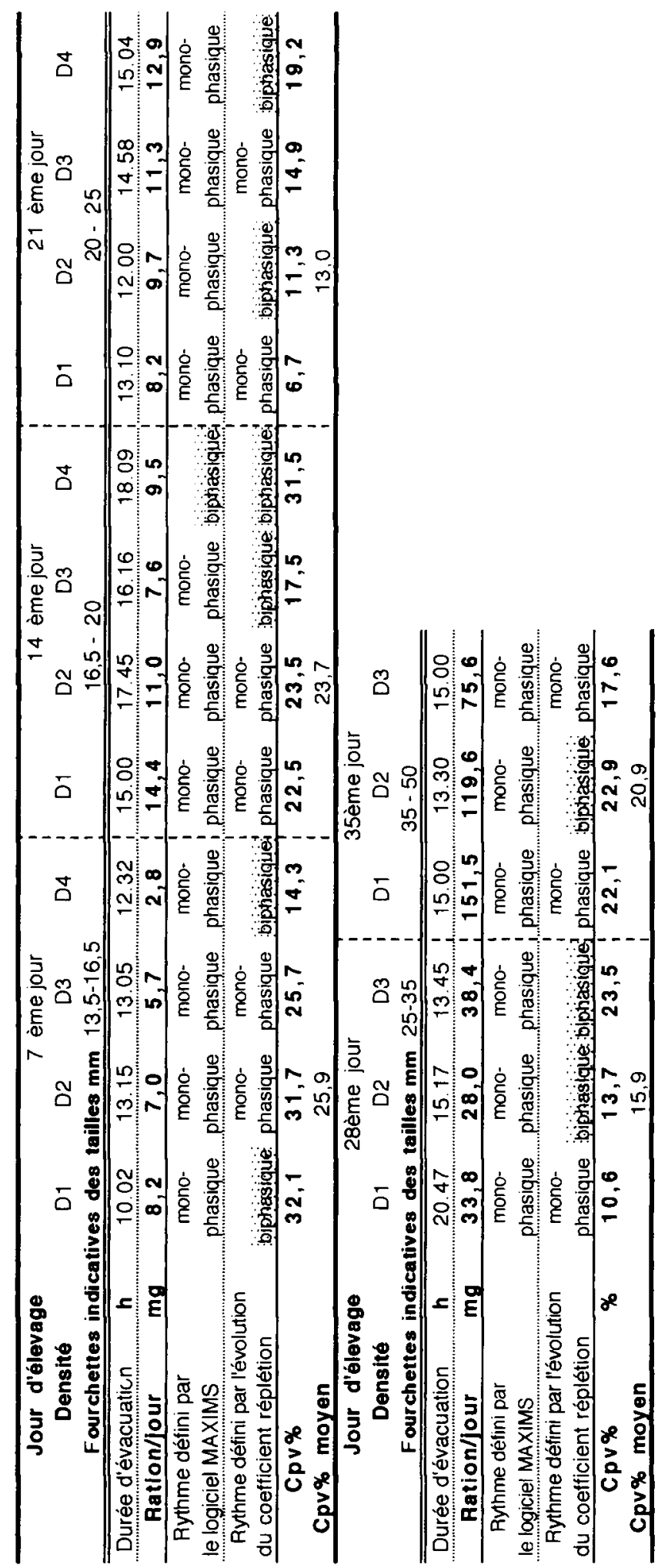


résultats. Les variabilités entre bacs de même densité sont difficilement explicables, compte tenu de l'homogénéité des facteurs environnementaux et des conditions d'alimentation. Cet "effet bassin ", déjà signalé par BRY (1992), pourrait être attribué aux différences d'apprentissage alimentaire (conditionnant la survie ultérieure) entre lots de brochets (IVANOVA et LOPATKO, 1983).

Sachant que les fractions inférieures à $150 \mu \mathrm{m}$, comprenant les Rotifères ou les Protozoaires, sont peu fréquemment consommées naturellement lors de la première prise de nourriture des larves de brochet, selon BALVAY (1983) et BRY, ISRAEL et MOYAT (1992), et que les taux de survie à 8 jours de ces larves sont bien supérieurs pour des proies de taille comprise entre 150 et $450 \mu \mathrm{m}$ (BRY, ISRAEL et MOYAT, 1992), nous avions choisi de tamiser le zooplancton récolté directement entre 300 et $500 \mu \mathrm{m}$ et nous confirmons que le démarrage des larves fonctionne très bien (bonne croissance, faible mortalité, GRES, 1994). Nos observations directes et celles de BRY, ISRAEL et MOYAT (1992) indiquent que les larves de brochet, au stade de la première alimentation exogène, consomment effectivement des Rotifères de petite taille (comprise entre 80 et $250 \mu \mathrm{m}$ ), mais ceci uniquement si les Rotifères constituent la seule ressource alimentaire. Dès l'introduction de petits Cladocères $(300-500 \mu \mathrm{m})$, les Rotifères sont délaissés.

Les tailles moyennes des poissons obtenus à 35 jours ( $L T=35$ à $50 \mathrm{~mm}$ ) sont légèrement inférieures ou comparables à celles rapportées dans les expériences antérieures d'élevage intensif des brochets sur proies vivantes zooplanctoniques (tableau VI). Ainsi, ARRIGNON (1976) enregistrait, à $15^{\circ} \mathrm{C}$ et au bout de 35 jours d'élevage, une longueur comprise entre 50 et $60 \mathrm{~mm}$ pour une densité initiale de $5000 \mathrm{VR} / \mathrm{m}^{3}$. HUET (1976) observait une longueur totale comprise entre 30 et $50 \mathrm{~mm}$ pour des sujets âgés de 28 à 42 jours, alimentés en plancton à $15^{\circ} \mathrm{C}$, avec une mise en charge initiale comprise entre 5000 et $25000 \mathrm{VR} / \mathrm{m}^{3}$. COCHE et BIANCHI (1979) donnaient une longueur comprise entre 25 et $40 \mathrm{~mm}$ pour des sujets âgés de 14 à 21 jours, sous un régime thermique de 10 à $14^{\circ} \mathrm{C}$ et une mise en charge initiale de l'ordre de 80 à $100000 \mathrm{VR} / \mathrm{m}^{3}$ (avec, toutefois, un taux de survie très faible : 10-20\%). GUERRIN (1983), à propos de l'élevage intensif de brochetons en Hollande, rapportait un taux de survie compris entre 50 et $90 \%$ sur zooplancton et une taille moyenne comprise entre 35 et $50 \mathrm{~mm}$, au bout de 24 jours à $15^{\circ} \mathrm{C}$, pour des densités initiales de $12000 \mathrm{VR} / \mathrm{m}^{3}$.

La croissance étant fortement dépendante de la température (HAKKARI et BAGGE, 1985 ; BRY et al., 1991), les plus faibles tailles obtenues ici par rapport aux expériences antérieures sont à mettre en relation avec la baisse de température accidentelle de la troisième semaine. En absence d'incident, en 1992, 75000 brochetons ont été produits à l'Institut Aquapure $(70 \mathrm{~mm}$ de longueur moyenne et un poids moyen de $2,5 \mathrm{~g}$ ) en 42 jours, à une densité finale de 5200 poissons $/ \mathrm{m}^{3}$ pour $40 \%$ de taux de survie moyen, et 235000 individus en $1993(50-60 \mathrm{~mm}$; 0,8 à $1,2 \mathrm{~g}$ ) en 30 jours, pour une densité finale de 12000 brochetons $/ \mathrm{m}^{3}$ et un taux de survie de $50 \%$ (GRES, 1994).

L'élevage intensif n'est donc pas incompatible avec une croissance satisfaisante, pourvu que l'alimentation soit optimisée, même si la croissance du brochet en système intensif est un peu inférieure à celle observée en étangs. Les coefficients spécifiques de croissance (ici : 9,9 à $11,2 \%$, de 11 à $50 \mathrm{~mm} \mathrm{LT}$ à $14,5^{\circ} \mathrm{C}$ ) sont inférieurs ou comparables à ceux enregistrés en milieu naturel $(11,9 \%$, FRANKLIN et SMITH, 1963 : entre 12 et $75 \mathrm{~mm}$ LT à une température de 12 à $21^{\circ} \mathrm{C} ; 11,4 \%$, LEJOLIVET, 1988 : entre 12 et $25 \mathrm{~mm} \mathrm{LT}$, pour une température de 12 à $15^{\circ} \mathrm{C}$; $14,9 \%$, BRY et al., 1991 : entre 34 et $54 \mathrm{~mm} \mathrm{LT} \mathrm{;} \mathrm{15,3 \% ,} \mathrm{DESVILETTES} \mathrm{et} \mathrm{al.,} 1994$ : entre 11 et $46 \mathrm{~mm} \mathrm{LT}$, sans indication sur la température d'élevage).

La densité a un effet déterminant sur la croissance des brochetons : on observe une diminution du coefficient spécifique de croissance avec l'augmentation de la mise en charge, la meilleure croissance étant enregistrée pour la plus faible mise en charge initiale $\left(5000 \mathrm{VR} / \mathrm{m}^{3}\right)$.

\section{Effet du cannibalisme}

Le cannibalisme débute précocement dès la deuxième semaine d'élevage (taille minimale $20 \mathrm{~mm}$ ), ce qui rejoint certains résultats rapportés antérieurement $(22 \mathrm{~mm}$ en aquarium, DORIER, 
Tableau VI : Données de la littérature sur la survie (\%) et la relation âge (jours) - longueur totale (mm) des brochetons en conditions naturelles, semi-naturelles et en élevage intensif.

Table VI : Literature data on early survival (\%) and on relationship between age (days) and total length $(\mathrm{mm})$ of young pikes in natural environments, in rearing ponds and under intensive culture conditions.

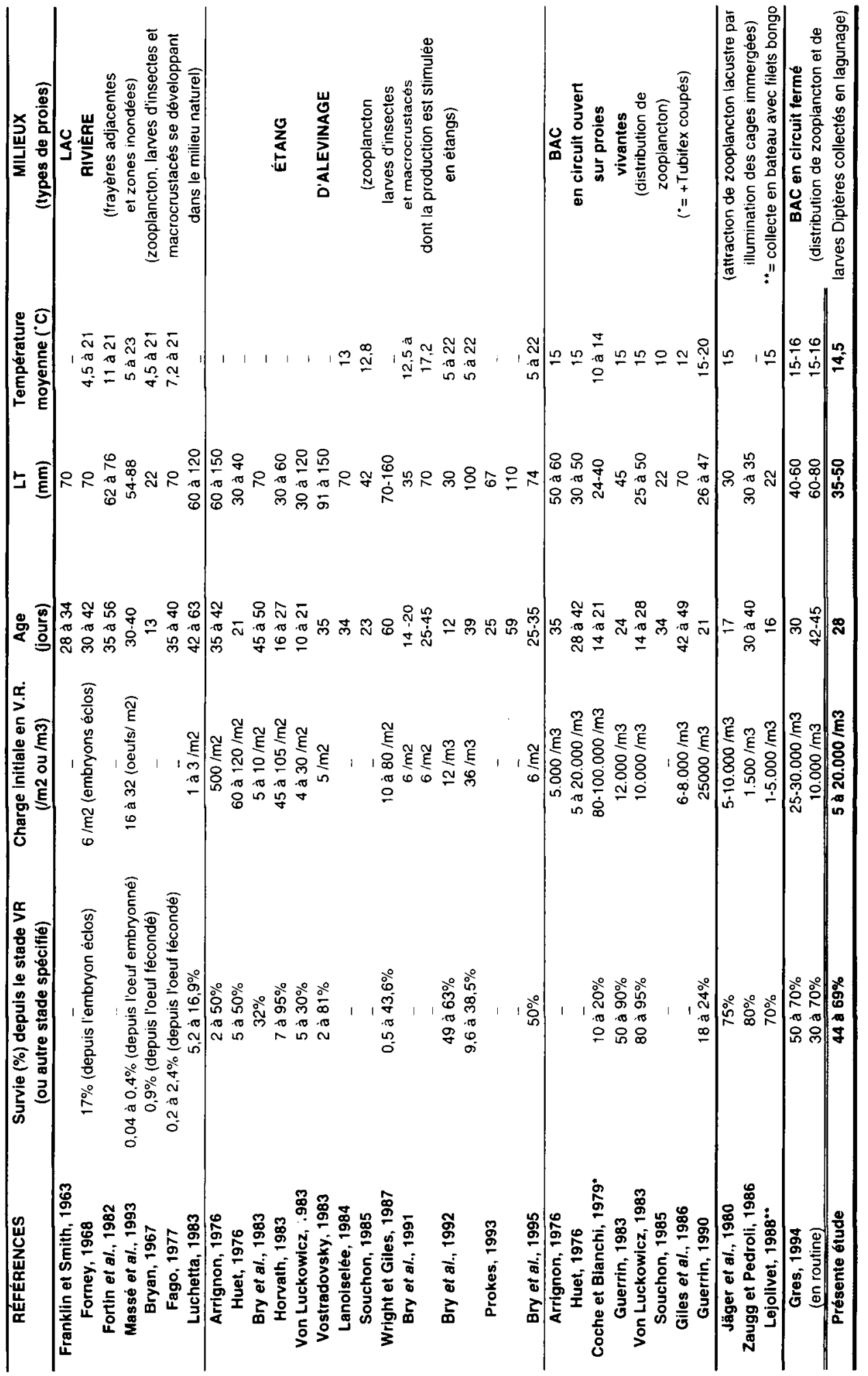


$1938 ; 21 \mathrm{~mm}$ sur une frayère, HUNT et CARBINE, $1951 ; 20 \mathrm{~mm}$ en cage flottante, LEJOLIVET, 1988). Cependant, ce cannibalisme précoce ne s'exerce que sur les poissons malingres (" pinhead fry") non adaptés à l'élevage (PECOR, 1978 ; LAPLANTE, 1988), et peut être stoppé par l'introduction de larves de Chaoboridés et la réalisation d'un tri des poissons par classes de tailles (GRES et BELAUD, 1995).

Au stade 28 jours ( $45 \mathrm{~mm}, \mathrm{LT}$ ), la proportion de poissons dévorés est comprise entre 8 et $29 \%$. II n'existe pas de chiffres de comparaison dans les travaux traitant de l'élevage intensif (ARRIGNON, 1976 ; COCHE et BIANCHI, 1979 ; GUERRIN, 1983) ; ces auteurs signalant seulement que le cannibalisme est limité pour l'obtention du stade $45 \mathrm{~mm}$ (LT). Nos résultats ne sont également pas comparables aux résultats obtenus par BRY et al. (1992), qui enregistrent entre 8 et $42 \%$ de poissons cannibales (et non pas victimes du cannibalisme) au bout de 39 jours $(100 \mathrm{~mm}, \mathrm{LT})$ d'élevage en petits étangs d'alevinage (mise en charge initiale 12 à $36 \mathrm{VR} / \mathrm{m}^{3}$ ); le cannibalisme ne s'instaurant qu'au-delà de $60 \mathrm{~mm}$ (LT).

L'importance du cannibalisme dans notre étude augmente avec la densité, conformément à ce qu'ont constaté GILES et al. (1986) et BRY et al. (1992). En aquarium, et sous alimentation zooplanctonique, GILES et al. (1986) enregistrent seulement $4 \%$ de mortalité par cannibalisme, à l'issue de 7 semaines d'élevage de lots de brochets élevés à $12{ }^{\circ} \mathrm{C}$ à partir du début de l'alimentation exogène. Ce cannibalisme, très inférieur à celui de la présente expérimentation, pourrait s'expliquer par les mises en charge initiales ( 280 à $\left.830 \mathrm{VR} / \mathrm{m}^{2}\right)$ inférieures aux nôtres (1560 à $6250 \mathrm{VR} / \mathrm{m}^{2}$ ) et par la plus faible température appliquée. En effet, on peut admettre les hypothèses, d'une part, d'une augmentation " comportementale " du cannibalisme lorsque la probabilité de rencontre entre brochets augmente avec la densité (GRES et BELAUD, 1995) et, d'autre part, d'une augmentation « métabolique » des besoins énergétiques quand la température augmente (BRY et GILLET, 1980 ; CRAIG et KIPLING, 1983).

\section{Influence de la taille et du poids des poissons sur la consommation journalière de proies}

S'il n'y a pas d'effet de la densité des larves de brochet sur le choix des proies, nous confirmons que ce choix est fonction de la taille des brochets. Nous avions déjà observé des corrélations positives hautement significatives entre la taille des brochets et celles des proies ingérées $(r=0,87 ; p=0,001 ; 144 \mathrm{ddl}$, pour Daphnia pulex et $r=0,74 ; p=0,001 ; 86 \mathrm{ddl}$, pour les larves de Chironomidés ; GRES, 1995). Au fur et à mesure de la croissance, la taille moyenne des proies augmente et la part des Chaoboridae devient prépondérante. Les proportions de proies zooplanctoniques dans les estomacs sont comparables, dans la majorité des cas, à celles rencontrées dans le milieu d'élevage. Ceci confirme le caractère alimentaire opportuniste des brochets (GRES, 1994 ; GRES, 1995), la sélection s'exerçant plutôt aux dépens des tailles de proies que sur leur appartenance taxonomique (FROST, 1954 ; FRANKLIN et SMITH, 1963 ; BALVAY, 1983 ; MANELPHE, 1989).

Dans les présentes conditions d'élevage, la taille du poisson a plus d'influence que la densité de mise en charge des bacs sur la consommation totale journalière. Ceci diffère des résultats de LEJOLIVET (1988), qui a observé un rôle de la densité initiale de brochetons élevés en cage sur la consommation journalière. Cette différence serait attribuable à une plus grande compétition, car les quantités de zooplancton distribuées par LEJOLIVET (1988) (175 g en 16 jours $/ 1000$ poissons) étaient 3,5 fois moins importantes que celles distribuées dans le cas présent (597 g en 16 jours/1000 poissons). Ainsi, il apparaît qu'une distribution abondante de nourriture puisse gommer les compétitions alimentaires dans les conditions de densité testées. Le couplage de l'élevage à une lagune produisant en abondance des proies de taille adéquate s'avère donc indispensable pour envisager un élevage intensif de jeunes brochets économiquement viable (GRES, 1994). Le lagunage peut être considéré, à ce titre, comme " un lieu de production de la matière première nécessaire à l'alimentation de l'élevage "(GUERRIN, 1990). 
La quantité journalière de nourriture ingérée $(\mathrm{Rj})$ augmente régulièrement avec le poids du poisson, et ceci indépendamment de la densité. La ration rapportée au poids vif du brochet (Cpv \%) est comprise entre 6 et $30 \%$, valeurs comparables à celles indiquées par SMISEK (1968), LUQUET et LUQUET (1983) et LEJOLIVET (1988), 5 à $36 \%$ pour des poissons de gamme de taille 13 à $60 \mathrm{~mm}$ (LT). LUQUET et LUQUET (1983) observent que le $\mathrm{Cpv} \%$ diminue régulièrement avec la taille du poisson, du premier stade de nutrition (VR) jusqu'à la taille $44 \mathrm{~mm}$, LT (soit $420 \mathrm{mg}$, Pt). La forte chute momentanée au stade $20-25 \mathrm{~mm}$, apparue dans cette étude, est concomitante des changements de température de l'eau de renouvellement. En faisant abstraction de cet artefact, nous considerons que, globalement, le taux de consommation journalier diminue au cours de la croissance.

Si la densité d'élevage n' apparaît pas intervenir globalement sur le taux de consommation de nourriture, elle joue cependant un rôle dans le comportement alimentaire. Pour les différents stades de brochets observés, on constate que plus la densité augmente, plus la proportion de zooplancton est forte dans les estomacs par rapport aux larves de Chaoboridés. Ceci pourrait traduire un manque de Chaoboridés dans les lots élevés aux plus fortes densités et on pourrait recommander d'accroître la part de ces proies, dont la taille apparaît particulièrement adéquate pour l'alimentation des jeunes brochets à partir de la troisième semaine d'élevage.

\section{CONCLUSION}

La production en masse de brochetons au moyen de proies vivantes facilement disponibles en bassins de lagunage naturel (GRES, 1994) peut constituer l'une des voies permettant d'assurer les repeuplements dans les milieux naturels (où se pose un problème de recrutement) et de satisfaire la demande accrue des pêcheurs sportifs. Et ceci, tant que les essais d'alimentation des larves de brochet sur aliment artificiel n'auront pas débouché vers des perspectives satisfaisantes sur les plans biologique et économique (GRAFF et SORENSON, 1970 ; GRAFF, 1978 ; LUQUET et LUQUET, 1983 ; KAUSHIK et al., 1985 ; BENDER et GRAFF, 1986).

Le rationnement journalier en proies vivantes appliqué ici peut être utilisé comme base par les ésociculteurs. La méthode d'élevage, décrite en détail dans GRES (1994), est facilement transposable dans les régions où il existe un parc important de stations de lagunage. De plus, les techniques et matériels efficaces de collectes actives (BARNABÉ, 1980 et 1989 ; GRES, 1994) peuvent permettre d'exploiter les biomasses d'invertébrés planctoniques dans de nombreuses retenues collinaires (Massif central, Sud-Ouest).

D'un point de vue dimensionnement des installations ésocicoles intensives, un circuit fermé comprenant $24 \mathrm{~m}^{2}$ de bacs (soit 6 unités de $4 \mathrm{~m}^{2}$ ) peut permettre de produire 200 à 250000 brochetons (40-60 mm, LT) par an, à raison de deux à trois phases de grossissement printanières pour une densité finale de 2 à 3000 poissons $/ \mathrm{m}^{2}$; le seuil de rentabilité (après amortissement et imposition) se situant aux alentours de 225000 pièces/an (GRES, 1994).

\section{REMERCIEMENTS}

Les auteurs tiennent à remercier Monsieur Olivier MARVALIN, directeur de I'Institut de Recherche et de Développement Aquapure, pour nous avoir permis de réaliser ce travail et Monsieur Philippe CENNI, technicien piscicole, pour son aide et ses conseils lors de l'élevage, et tout particulièrement Monsieur Christian BRY (INRA), pour son aide précieuse lors de l'élaboration de cet article.

\section{BIBLIOGRAPHIE}

ARRIGNON J., 1976. L'Esociculture. In Aménagement des eaux continentales. Gauthier-Villars, 3e édition, Paris, 140-148. 
BALON E.K., 1975. Terminology of intervals in fish development. J. Fish. Res. Bd. Can., 32 , 1663-1670.

BALVAY G., 1983. L'alimentation naturelle des alevins de Brochet durant leur premier mois de vie. In BILLARD R. (ed.), Le brochet: Gestion dans le milieu naturel et élevage, 179-198, INRA Publi., Paris.

BARNABÉ G., 1979. Utilisation des chaînes alimentaires naturelles et du recyclage des eaux usées dans la production à grande échelle de juvéniles pour l'aquaculture. Publ. Sci. Tech. CNEXO : actes colloq., 7, 221-238.

BARNABÉ G., 1980. Système de collecte du zooplancton à l'aide de dispositifs autonomes et stationnaires. In BILLARD R., La Pisciculture en étangs, 429-434, INRA Publi., Paris.

BARNABÉ G., 1989. Les collectes de zooplancton. In BARNABÉ G. (ed.), Aquaculture, volume 1, 261-272, Tec \& Doc., Lavoisier.

BENDER T.R., GRAFF D.R., 1986. Pennsylvania's practices for intensive culture of hybrid muskellunge. Am. Fish. Soc. Spec. Publ., 15, 279-284.

BRAUM E., 1964. Experimentelle Untersuchungen zur ersten Nahrungsaufnahme und Biologie an Jungfischen von Blaufelchen (Coregonus wartmanni, Bloch), Weissfelchen (Coregonus fera, Jurine) und Hechten (Esox lucius, L.). Arch. Hydrobiol., suppl., 28 (2/3), 183-244.

BRY C., 1992. Reproduction contrôlée du brochet (Esox lucius) et dynamique des jeunes stades en petits étangs : aspects zootechniques et écologiques. Thèse UCBL, 292-92, $277 \mathrm{p}$.

BRY C., GILLET C., 1980. Reduction of cannibalism in pike fry (Esox lucius, Linnaeus) by isolation of full-sib families. Reprod. Nutr. Develop., 20 (1A), 173-182.

BRY C., SOUCHON Y., NEVEU G., TREBAOL L., 1983. Production de familles de brochetons en petits étangs par RNA : bilan de trois années d'expérimentation et comparaison avec la méthode d'alevinage. Bull. Fr. Piscic., 228, 46-56.

BRY C., HOLLEBECQ M.G., GINOT V., ISRAEL G., MANELPHE J., 1991. Growth patterns of pike (Esox lucius, Linnaeus) larvae and juveniles in small ponds under various natural temperature regimes. Aquaculture, 97, 155-168.

BRY C., BASSET E., ROGNON X., BONAMY F., 1992. Analysis of sibling cannibalism among pike, Esox lucius, juveniles reared under semi-natural conditions. Env. Biol. Fish., 35, 75-84.

BRY C., ISRAEL G., MOYAT P., 1992. Caractéristiques des premières proies de la larve de brochet (Esox lucius) : relation entre quantité de zooplancton initiale et survie ultérieure des jeunes poissons en étang. In BRY C., Thèse UCBL, 292-92, $277 \mathrm{p.}$

BRY C., BONAMY F., MANELPHE J., DURANTHON B., 1995. Early life characteristics of pike, Esox lucius, in rearing ponds : temporal survival pattern and ontogenic diet shifts. J. Fish. Biol., 46, 99-113.

BRYAN J.E., 1967. Northern pike production in Phalen pond, Minnesota. J. MN. Acad. Sci., 34, 101-109.

CHODOROWSKA W., CHODOROWSKI A., 1975. Substitution d'éléments de la diète chez les alevins de poissons carnassiers. Verh. Int. Ver. Limnol., 19 (2), 2556-2562.

COCHE A.G., BIANCHI G., 1979. Present status of mass rearing of fry and fingerlings in the EIFAC region. In HUISMAN E.A. and HOOGENDORN H., EIFAC, Tech. pap., 35, suppl. 1, 7-31.

CRAIG J.F., KIPLING C., 1983. Reproduction effort versus the environment : case histories of Windermere perch, Perca fluviatilis Linnaeus, and pike, Esox lucius Linnaeus. J. Fish. Biol., 22, 713-727. 
CROSSMAN E.J., CASSELMAN J.M., 1987. An annoted bibliography of the pike Esox lucius (Osteichthyes, Salmoniformes). Toronto, Royal Ontario Museum Publications in Life Sciences, $386 \mathrm{p}$.

CULVER D.A., BOUCHERLE M.M., DEAN D., FLETCHER J.W., 1985. Biomass of freshwater crustacean zooplankton from length-weight regressions. Can. J. Fish. Aquat. Sci., 42, $1380-1390$.

DESVILETTES C., BOURDIER G., BRETON J.C., 1994. Lipid class and fatty acid composition of planktivorous larval pike Esox lucius living in a natural pond. Aquat. Living Resources, 7 , $67-77$.

DORIER A., 1938. A propos de l'oeuf et de l'alevin de brochet. Bull. Fr. Piscic., 110, 61-73.

DROST M.R., 1987. Relation between aiming and catch success in larval fishes. Can. J. Fish. Aquat. Sci., 44, 304-315.

DUMONT H.J., VAN DE VELDE I., DUMONT S., 1975. The dry weight estimation of biomass in a selection of Cladocera, Copepoda and Rotifera from the plankton, periphyton and benthos of continental waters. Oecologia, 19, 75-97.

DUMONT H.J., BALVAY G., 1979. The dry weight estimate of Chaoborus flavicans (Meigen) as a function of length and instars. Hydrobiologia, 64, 139-145.

DURIEU M., 1995. Cycle biologique de Chaoborus flavicans (Diptera, Chaoboridae), valorisation de la production de biomasse en bassin de lagunage et optimisation en conditions expérimentales. Thèse UPS Toulouse, $303 p$.

FAGO D.M., 1977. Northern pike production in managed spawning and rearing marshes. Wis. Dep. Nat. resources, Tech. Bull., 96, $30 \mathrm{p}$.

FORNEY J.L., 1968. Production of young northern pike in a regulated marsh. N.Y. Fish Game, 15 , 143-154.

FORTIN R., DUMONT P., FOURNIER H., CADIEUX C., VILLENEUVE D., 1982. Reproduction et forces des classes d'âge du grand brochet (Esox lucius, Linnaeus) dans le Haut Richelieu et la baie du Missisquoi. Can. J. Zool., 60, 227-240.

FRANKLIN D.R., SMITH L.L., 1963. Early life history of the northern pike, Esox lucius L., with special reference to the factors influencing the numerical strength of year classes. Trans. Am. Fish. Soc., 92, 91-110.

FROST W.E., 1954. The food of pike in Windermere. J. Anim. Ecol., 23, 339-360.

FROST W.E., KIPLING C., 1967. A study of reproduction, early life, weight-length relationship and growth of pike, Esox lucius, in Windermere. J. Anim. Ecol., 36, 651-693.

GILES N., WRIGHT R.M., NORD M.E., 1986. Cannibalism in pike fry, Esox lucius L. : some experiments with fry densities. J. Fish Biol., 29, 107-113.

GRAFF D.R., 1978. Intensive culture of Esocids. The current state of the art. Am. Fish. Soc. Spec. Publ., 11, 195-201.

GRAFF D.R., SORENSON L., 1970. The successful feeding of a dry diet to Esocids. Prog. Fish. Cult., 32, 31-35.

GRES P., 1994. Production intensive de brochetons (Esox lucius, L.) nourris de proies vivantes issues de bassins de lagunage naturel : survie, croissance, alimentation. Thèse UBP Clermont-Ferrand, 627-67, 247 p. + 58 p. annexes.

GRES P., 1995. Aspects quantitatifs de l'alimentation zooplanctonophage et entomophage de brochets (Esox lucius, L.) en élevage intensif. Ichtyophysiologica Acta, 18, 1-21.

GRES P., BELAUD A., 1995. Limitation du cannibalisme précoce des brochetons (Esox /ucius, L.) en élevage intensif. Ichtyophysiologica Acta, 18, 23-41. 
GUERRIN F., 1983. Elevage intensif de brochetons et d'alevins de repeuplement aux Pays-Bas. Rapport CEMAGREF, Montpellier, $31 \mathrm{p}$.

GUERRIN F., 1990. Valorisation aquacole d'eaux usées traitées par lagunage naturel. Évaluation biotechnique et modélisation des connaissances. Thèse UPS, $297 \mathrm{p}$.

HAKKARI L.R., BAGGE P., 1985. On the fry density of pike (Esox lucius, L.) in lake Saiman, Finland. Verh. Internat. Verein. Limnol., 22, 2560-2565.

HORVATH L., 1983. Elevage et production du brochet (Esox lucius, L.) en Hongrie. In BILLARD R. (ed.), Le brochet : Gestion dans le milieu naturel et élevage, 215-223, INRA Publi., Paris.

HUET M., 1976. Reproduction, incubation et alevinage du brochet. EIFAC, Tech. Pap., 25, 147-163.

HUNT B.P., CARBINE W.F., 1951. Food of young pike, Esox lucius L., and associated fishes in Peterson's ditches, Hougthon Lake, Michigan. Trans. Am. Fish. Soc., 80, 67-83.

IVANOVA M.N., LOPATKO A.M., 1983. Feeding behavior of pike, Esox lucius (Esocidae), larvae from the progeny of a single pair of spawners. J. Ichtyol., 23 (4), 171-173.

IVLEV V.S., 1961. Experimental ecology of the feeding of fishes. Yales Univ. Press, New Haven, $302 \mathrm{p}$.

JÄGER T., DAUSTER H., KIWUS A., 1980. Aufzaucht von Hechtsetzlingen in erleuchteten Netzgehegen. Fischer und Teichwirt, 31, 323-326.

JARRE A., PALOMARES M.L., SORIANO M.L., SAMBILAY Jr., PAULY D., 1990. A user's manual for MAXIMS. A computer program for estimating the food consumption of fishes from diel stomach contents data and population parameters. ICLARM Software 4, International Centre for Living Aquatic Resources Management, Manila, Philippines, 27.

KAUSHIK S.J., DABROWSKI K., LUQUET P., 1985. Experimental studies on some trophic relationships in juvenile pike, Esox lucius, L. J. Fish Biol., 26, 171-188.

KINKELIN (DE) P., MICHEL C., GHITTINO P., 1985. Précis de pathologie des poissons. INRA OIE, $348 \mathrm{p}$

KLUPP R., 1978. Die Produktion von Hechtsetzlingen in Karpfenteichen. Fischer und Teichwirt, $29,144-148$.

LANE T.H., JACKSON H.M., 1969. Voidance time for 23 species of fish. Invest. Fish. Cont., 33, 9 p.

LANOISELÉE B., 1984. Fertilisation organique en aquaculture : utilisation du lisier de porc pour l'alevinage de poissons d'étangs. Thèse Docteur - Ingénieur, I.N.A., Paris Grignon, 252 p.

LAPLANTE G., 1988. L'élevage du maskinonge (Esox masquinongy) en circuit fermé et à la diète sèche : données techniques. Ministère du loisir, de la chasse et de la pêche du Québec. Service de l'aménagement et de l'exploitation de la faune de l'Estrie. Station piscicole de Baldwin, Sherbrook, $45 \mathrm{p}$.

LAUZANNE L., 1975. Régimes alimentaires d'Hydrocyon forskalii (Pisces, Characidae) dans le lac Tchad et ses tributaires. Cah. ORSTOM, Ser. Hydrobiol., 9, 105-121.

LEJOLIVET C., 1988. Contribution à l'étude du recrutement des poissons du réservoir de Pareloup. Élevage et étude de la phase planctonophage des brochetons (Esox lucius, L.). Régime alimentaire, croissance, répartition spatio-temporelle de la communauté alevinique en zone littorale. Thèse INP, Toulouse, 288 p. +61 p. d'annexes.

LUCHETTA J.C., 1983. Utilisation de sites naturels pour le grossissement d'alevins de brochet produits en écloserie. In BILLARD R. (ed.), Le brochet : Gestion dans le milieu naturel et élevage, 189-208, INRA Publi., Paris.

LUQUET P., LUQUET J.F., 1983. Appréciation du niveau d'ingestion et de la vitesse du transit alimentaire chez l'alevin de brochet nourri avec un aliment composé. In BILLARD R. (ed.), Le brochet : Gestion dans le milieu naturel et élevage, 235-243, INRA Publi., Paris. 
MANELPHE J., 1989. Reproduction naturelle aménagée du brochet (Esox lucius, L.) en petits étangs : suivi biologique et aspects économiques de la production de juvéniles. Thèse INP, Toulouse, 174 p. + annexes.

MASSÉ G., DUMONT P., FORTIN R., 1993. Survie des oeufs et juveniles et forces des classes d'âge du grand brochet (Esox lucius, L.) de la rivière aux Pins, Montréal, Québec. Can. J. Zool., 71, 368-375.

NiKOLSKY G.V., 1963. The ecology of fishes. Acad. press, London and New York, $352 \mathrm{p.}$

PALOMARES M.L., 1991. La consommation de nourriture chez les poissons : étude comparative, mise au point d'un modèle prédictif et application à l'étude des réseaux trophiques. Thèse INP, Toulouse, 211 p. + annexes.

PECOR C.H., 1978. Intensive culture of tiger muskellunge in Michigan during 1976 and 1977. Am. Fish. Soc. Spec. Publ., 11, 202-209.

PROKES M., 1993. Growth of pike (Esox lucius, L.) larvae and juveniles in the Musôv reservoir. Folia Zool., 42 (1), 77-93.

RAAT A.J.P., 1988. Synopsis of biological data on the northern pike, Esox lucius Linnaeus. F.A.O., Fish. synopsis, 30 (rev. 2), $178 \mathrm{p}$.

SMISEK J., 1968. The intensity of the acceptance of food by pike fry in the course of 24 hours. Bull. Vur. Vodnany, 3, 14-18.

SOUCHON Y., 1985. Reproduction du brochet (Esox lucius, L.) et développement des brochetons en dombes : éléments d'optimisation des modes de gestion extensive. Thèse Docteur Ingénieur, UCB, Lyon, $333 \mathrm{p}$.

VON LUCKOWICZ M., 1983. Production et élevage du brochet en République fédérale allemande. In BILLARD R. (ed.), Le brochet: Gestion dans le milieu naturel et élevage, 225-234, INRA Publi., Paris.

VOSTRADOVSKY J., 1983. Techniques et méthodes d'aménagement et d'élevage du brochet en Tchécoslovaquie. In BILLARD R. (ed.), Le brochet : Gestion dans le milieu naturel et élevage, 271-281, INRA Publi., Paris.

WRIGHT R.M., GILES N., 1987. The survival, growth and diet of pike fry, Esox lucius L., stocked at different densities in experimental ponds. J. Fish Biol., 30, 617-629.

ZAUGG B., PEDROLI J.C., 1986. The rearing of young fish in illuminated net cages in lake Neuchâtel, Switzerland. Arch. Hydrobiol. Beih. Ergebn. Limnol., 22, 215-230. 